\title{
Article \\ No Influence of Previous Coxiella burnetii Infection on ICU Admission and Mortality in Emergency Department Patients Infected with SARS-CoV-2
}

\author{
Jesper M. Weehuizen 1,*(D), Rik van Spronsen ${ }^{1}$, Andy I. M. Hoepelman ${ }^{1}$, Chantal P. Bleeker-Rovers ${ }^{2}$, \\ Jan Jelrik Oosterheert ${ }^{1}$ (D) and Peter C. Wever ${ }^{3}$
}

\section{check for}

updates

Citation: Weehuizen, J.M.; van Spronsen, R.; Hoepelman, A.I.M.; Bleeker-Rovers, C.P.; Oosterheert, J.J.; Wever, P.C. No Influence of Previous Coxiella burnetii Infection on ICU Admission and Mortality in Emergency Department Patients Infected with SARS-CoV-2. J. Clin. Med. 2022, 11, 526. https:// doi.org/10.3390/jcm11030526

Academic Editor: David Barnes

Received: 23 December 2021

Accepted: 17 January 2022

Published: 20 January 2022

Publisher's Note: MDPI stays neutral with regard to jurisdictional claims in published maps and institutional affiliations.

Copyright: (C) 2022 by the authors. Licensee MDPI, Basel, Switzerland. This article is an open access article distributed under the terms and conditions of the Creative Commons Attribution (CC BY) license (https:// creativecommons.org/licenses/by/ $4.0 /)$
1 Department of Internal Medicine and Infectious Diseases, University Medical Center Utrecht, 3584 CX Utrecht, The Netherlands; rikvanspronsen123@gmail.com (R.v.S.); I.M.Hoepelman-2@umcutrecht.nl (A.I.M.H.); J.J.Oosterheert@umcutrecht.nl (J.J.O.)

2 Department of Internal Medicine and Infectious Diseases, Radboud Expertise Center for Q Fever, Radboud University Medical Center, 6525 GA Nijmegen, The Netherlands; Chantal.Bleeker-Rovers@radboudumc.nl

3 Department of Medical Microbiology and Infection Control, Jeroen Bosch Hospital, 5223 GZ 's-Hertogenbosch, The Netherlands; P.Wever@jbz.nl

* Correspondence: j.m.weehuizen-2@umcutrecht.nl

\begin{abstract}
Background: the geographical similarities of the Dutch 2007-2010 Q fever outbreak and the start of the 2020 coronavirus disease 19 (COVID-19) outbreak in the Netherlands raised questions and provided a unique opportunity to study an association between Coxiella burnetii infection and the outcome following SARS-CoV-2 infection. Methods: We performed a retrospective cohort study in two Dutch hospitals. We assessed evidence of previous C. burnetii infection in COVID-19 patients diagnosed at the ED during the first COVID-19 wave and compared a combined outcome of inhospital mortality and intensive care unit (ICU) admission using adjusted odds ratios (OR). Results: In total, 629 patients were included with a mean age of 68.0 years. Evidence of previous C. burnetii infection was found in 117 patients (18.6\%). The combined primary outcome occurred in $40.2 \%$ and $40.4 \%$ of patients with and without evidence of previous C. burnetii infection respectively (adjusted OR of 0.926 (95\% CI 0.605-1.416)). The adjusted OR of the secondary outcomes in-hospital mortality, ICU-admission and regular ward admission did not show an association either. Conclusion: no influence of previous C. burnetii infection on the risk of ICU admission and/or mortality for patients with COVID-19 presenting at the ED was observed.
\end{abstract}

Keywords: Coxiella burnetii; Q fever; SARS-CoV-2; COVID-19; Dutch Q fever outbreak

\section{Introduction}

$\mathrm{Q}$ fever is a zoonosis, caused by the intracellular Gram-negative bacterium Coxiella burnetii. Infection occurs through inhalation of contaminated aerosols, derived from sheep, goats and cattle. The bacterium is very resistant to death by environmental factors and can be spread by the wind. Person-to-person transmission occurs rarely. In 60\% of humans, infection results in acute $Q$ fever, an influenza-like illness, sometimes accompanied with mild to severe pneumonia or hepatitis [1]. The Dutch Q fever outbreak occurred between 2007-2010 and was globally unique for its size and duration. Seroprevalence screening for C. burnetii revealed that around 40,000 people were infected around the epicenter of the outbreak [2]. Most infections were reported in the south-east of the Netherlands, due to a high density of goats $[2,3]$. In the years after the epidemic, focus shifted towards patients with long term effects of $Q$ fever suffering from $Q$ fever fatigue syndrome (QFS) or chronic $Q$ fever [4,5].

On 27 February 2020, the first patient in the Netherlands tested positive for the new severe acute respiratory syndrome coronavirus 2 (SARS-CoV-2) [6]. As the virus spread, the southern region of the Netherlands quickly became the epicenter of the Dutch coronavirus disease 2019 (COVID-19) outbreak [7]. Soon after, voices emerged that the epicenter of the 
COVID-19 outbreak showed striking geographical similarities with the epicenter of the Dutch Q fever outbreak. (Figure 1)
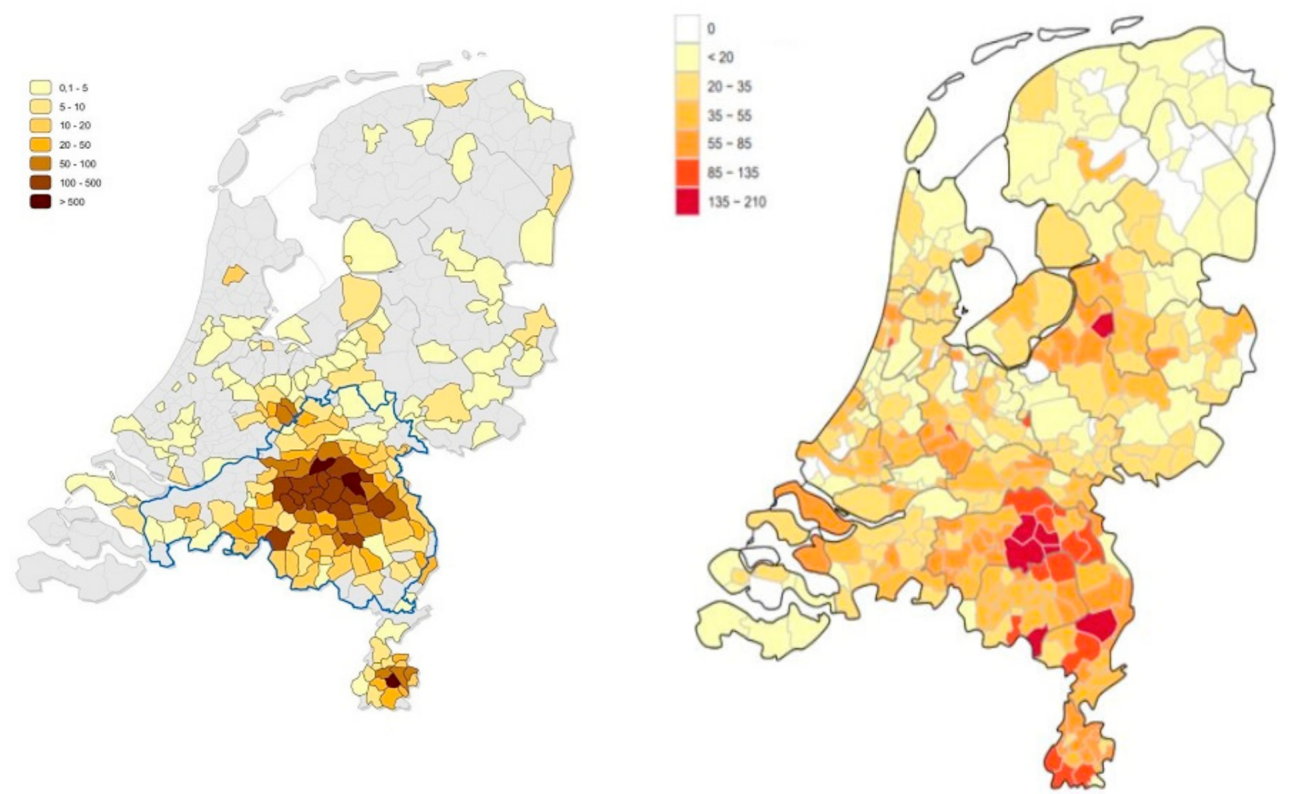

Figure 1. COVID-19 and $Q$ fever in the Netherlands. Left: number of reported $Q$ fever cases per 100,000 persons in 2009. Right: number of reported COVID-19 per 100,000 persons cases on 24 May 2020. Source: National Institute for Public Health and Environment (RIVM).

Immune dysregulation is linked to adverse clinical outcomes in COVID-19 patients [8]. The association between age, chronic diseases (e.g., diabetes mellitus and obesity) and severe SARS-CoV-2 infection are partially explained by immune dysregulation [8-11]. In addition, chronic or past infections, e.g., infections with Hepatitis B virus (HBV) and cytomegalovirus (CMV), are associated with immune dysregulation and potential adverse clinical outcomes in COVID-19 patients [12,13]. Earlier studies showed altered cytokine responses to viral ligands and lipopolysaccharide in $Q$ fever patients suffering from QFS. The mRNA expression of pro-inflammatory cytokines interleukin (IL)-1 $\beta$ and tumor necrosis factor (TNF)- $\alpha$ was decreased and an increased susceptibility to respiratory infections was suspected [14]. Another study demonstrated increased concentrations of anti-inflammatory cytokines IL-10 and IL-1Ra at six months after acute $Q$ fever infection. It was suggested that this was the result of altered transcriptional programming of myeloid cells [15]. As a result of this inflammatory dysregulation, $Q$ fever patients might be more susceptible to infections, e.g., with SARS-CoV-2, and may have an increased risk of symptomatic disease and hospitalization. On the other hand, it is also possible to hypothesize a protective effect after an infection with $C$. burnetii. It is suggested that induction of the innate immunity by Bacillus Calmette-Guerin (BCG) vaccination or previous infections could lead to protection against other (intracellular) pathogens [16,17]. BCG vaccination already showed protection against respiratory tract infections in elderly patients [18-20] and trials assessing the protection of BCG vaccination against SARS-CoV-2 are currently conducted [21-23].

The geographical similarities of the $Q$ fever outbreak and the start of the COVID-19 epidemic in the Netherlands raises questions and provided a unique opportunity to study an association between C. burnetii infection and the outcome following SARS-CoV-2 infection. As a possible consequence of this association, previous $C$. burnetii infection could be added as risk factor for severe SARS-CoV-2 infection with the appropriate preventive and therapeutic measure for these patients. The aim of this study is to assess the possible presence of an association between a previous $C$. burnetii infection and adverse outcome following SARS-CoV-2 infection. This association was studied in patients infected with 
SARS-CoV-2 during the first COVID-19 wave who presented at the emergency department (ED) of two hospitals located in the region highly affected by both the 2007-2010 Q fever epidemic and the first wave of the COVID-19 pandemic.

\section{Methods}

\subsection{Study Design}

We performed a retrospective cohort study in two Dutch hospitals. We assessed if there was evidence of a previous C. burnetii infection in adult patients ( $\geq 18$ years old) diagnosed with COVID-19, defined as a PCR-confirmed SARS-CoV-2 infection. Patients that were included had presented at the ED during the first COVID-19 wave between 23 February and 31 May 2020, in the Jeroen Bosch Hospital in 's-Hertogenbosch or Bernhoven in Uden. The primary outcome was a combined outcome of in-hospital mortality and intensive care unit (ICU) admission. Secondary outcomes were in-hospital mortality, ICU admission, and admission to a regular ward. We chose for a combined primary outcome because ICU admission in itself was affected by restriction in ICU admission due to lack of free ICU beds during the first wave of the pandemic. The combined outcome reflects bad outcome in COVID-19 patients and is not affected by this restriction.

\subsection{Evidence of Previous C. burnetii Infection}

Evidence of previous C. burnetii infection was assessed in the included COVID-19 patients. First, the electronic health records (EHR) were consulted. A previous positive result of $C$. burnetii PCR on serum, plasma or tissue, or a positive C. burnetii $\operatorname{IgM}$ or IgG antibody result was defined as a previous $C$. burnetii infection. A previous negative serology result obtained after the $Q$ fever pandemic, thus during or after 2011, was defined as no previous C. burnetii infection. If no information on previous C. burnetii infection could be obtained from the EHR, stored blood samples of these patients from regular clinical care or the "BioMarCo-19" study (BioMarCo-19-CMO 2020-6344) were assessed for C. burnetii antibody status. Phase I and Phase II IgG C. burnetii antibody titers were measured using indirect immunofluorescence assay (IFA) (Focus Diagnostics, Cypress, CA, USA). Titration was performed with binary serial dilutions, with a detection cut-off titer of 1:64.

\subsection{Data Collection}

Baseline characteristics, comorbidities including date of diagnosis, clinical data and outcome status of the included patients were obtained from the EHR. Comorbidities included cardiovascular disease, diabetes mellitus requiring medication, any (hematologic) malignancy, obstructive pulmonary diseases defined as asthma, chronic obstructive pulmonary disease (COPD), interstitial lung disease (ILD) or cystic fibrosis (CF) and immunocompromised state, defined as the use of corticosteroids (prednisone or equivalent, cumulative dose $>700 \mathrm{mg}$ ), anti-CD20 therapy, biologicals (TNF-alpha inhibitors, interleukin-5 inhibitors and monoclonal antibodies), methotrexate, azathioprine and / or mercaptopurine within the last six months, having received an autologous/allogenic stem-cell transplantation, having neutropenia $\left(<0.5 \times 10^{9} / \mathrm{L}\right)$, (functional) hypo/asplenia, CD4-penia $\left(<200\right.$ cells $\left./ \mathrm{mm}^{3}\right)$, hypogammaglobinemia and/or having a primary immunodeficiency. Furthermore, the updated Charlson comorbidity index (uCCI) was calculated [24]. Upon admission at the ED, vital parameters were documented, which we used to determine the modified early warning score (MEWS) [25].

\subsection{Statistical Analysis}

Data analysis was performed with SPSS (IBM SPSS Statistics for Windows, version 26.0.0.1. IBM Corp., Armonk, New York, USA). Figures were generated in SPSS and Microsoft Excel (Microsoft office for windows, Version 2013, Microsoft, Redmond, WA, USA). Multiple imputations were used to account for missing data on patient height and weight. We used both determinants, potential confounders and outcome variables in the imputation model and missing values were imputed under the assumption of missingness at random. Differences 
between patients with or without evidence of previous C. burnetii infection were assessed by univariate analysis using a Pearson's Chi-square test or Fisher's exact test for differences in proportions of binary variables and Mann-Whitney $U$ test or independent $t$-test for continuous variables, as appropriate. We compared the outcomes between patients with or without evidence of previous $C$. burnetii infection using adjusted odds ratios (OR) with a $95 \%$ confidence interval (CI) from multiple logistic regression. Age, sex and body mass index (BMI) were entered as confounders in the model. Comorbidities diagnosed before the end of the Q fever pandemic, before the start of 2011, were entered in the model if they were present in more than 10 patients.

\section{Results}

\subsection{Study Population}

During the first COVID-19 wave 1151 patients were diagnosed with COVID-19 in the Jeroen Bosch Hospital in 's-Hertogenbosch and Bernhoven in Uden. From 486 patients $(42.2 \%)$, no information on previous C. burnetii infection could be obtained (no previous C. burnetii PCR or serology results), and no stored blood samples were available. Of the remaining patients, 36 patients (3.1\%) were diagnosed with COVID-19 by serology only. These patients were also excluded from the analysis. Thus, in total, 629 patients (54.6\%) were included in this study (Figure 2). The number of patients with a positive SARS-CoV-2 PCR included per week is displayed in Figure 3.

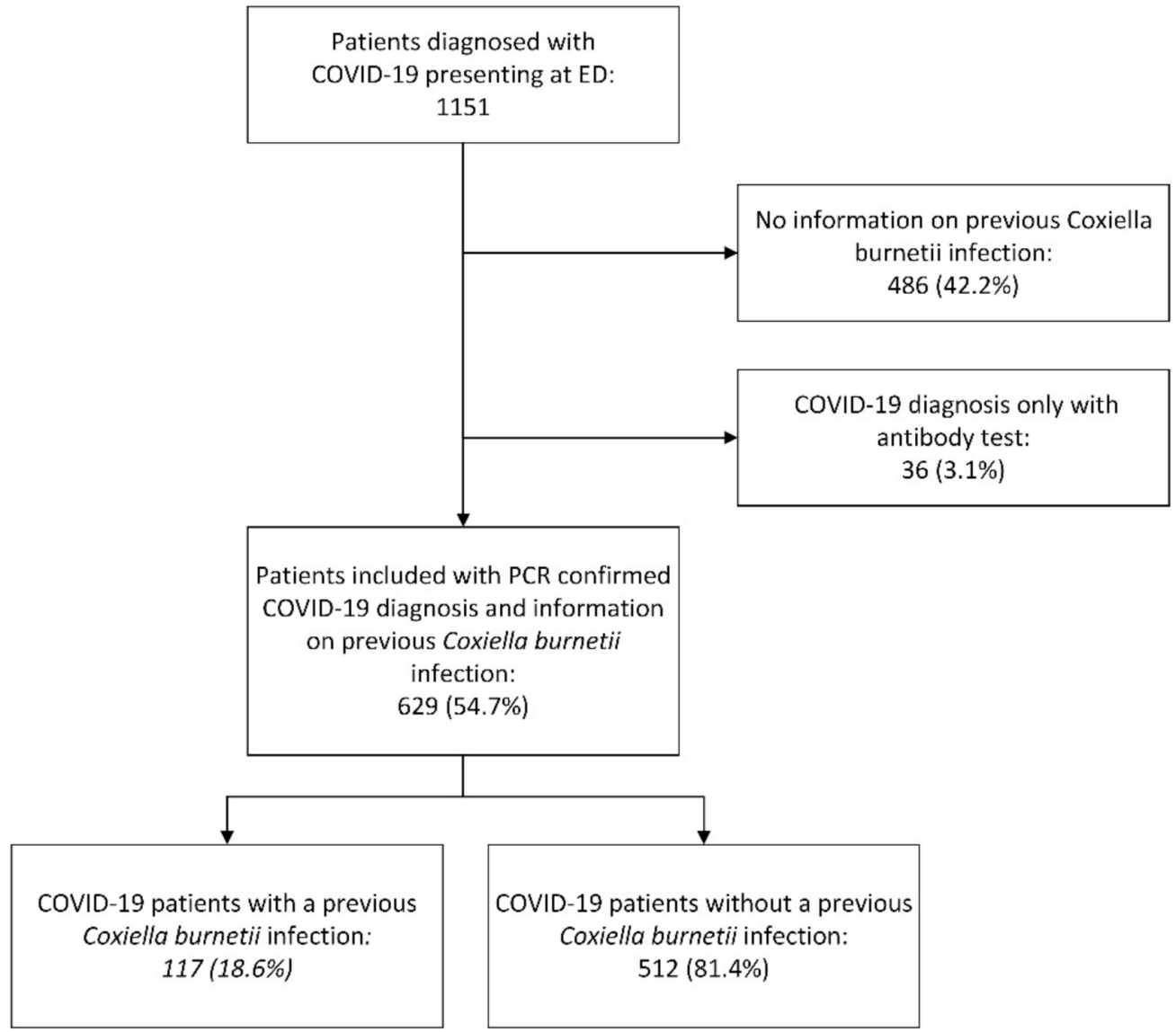

Figure 2. Flowchart of included patients. Abbreviations: COVID-19: coronavirus disease 19, ED: emergency department, PCR: polymerase chain reaction. 


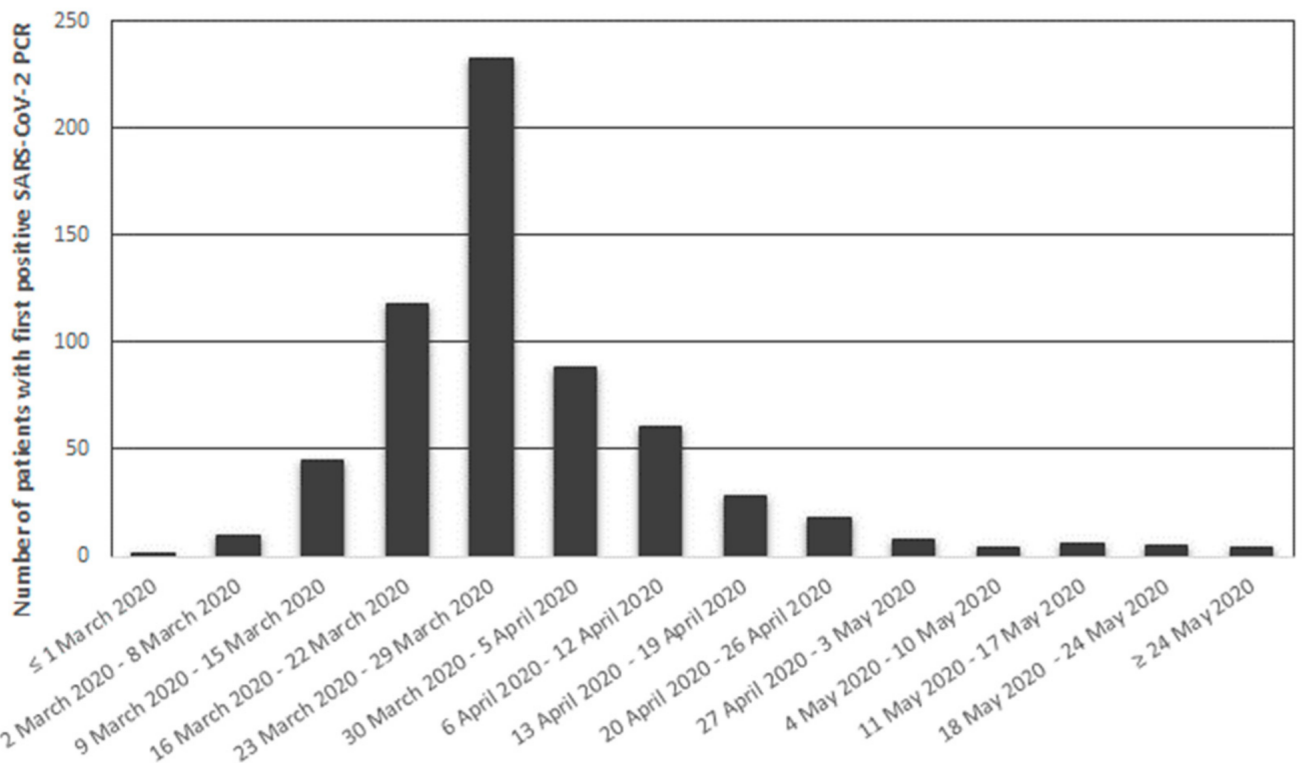

First COVID-19 wave seperated in weeks

Figure 3. Histogram of number of patients presenting at the Emergency Departments of the Jeroen Bosch Hospital and Bernhoven with a first positive SARS-CoV-2 PCR per week during the first COVID19 wave. Abbreviations: PCR: polymerase chain reaction, COVID-19: coronavirus disease 2019.

\subsection{Evidence of Previous C. burnetii Infection}

In the included patients, 117 patients $(18.6 \%)$ had evidence of previous C. burnetii infection while 512 patients $(81.4 \%$ ) had not (Figure 2). The information on previous C. burnetii infection was determined from earlier performed C. burnetii PCR and serology results extracted from the EHR in 133 patients (21.1\%). In these 133 patients, 52 patients (39.1\%) had evidence of previous C. burnetii infection while 81 patients $(60.9 \%)$ had no such evidence. In 496 patients (78.9\%), serology results were obtained through IFA performed on stored blood samples. In these patients, $65(13.1 \%)$ had evidence of previous C. burnetii infection while in 431 patients (86.9\%) serology showed no evidence of a previous infection.

\subsection{Baseline Characteristics}

Baseline characteristics are shown in Table 1. Mean age was 68.0 years $(\mathrm{SD} \pm 12.5$, with a mean $\mathrm{BMI}$ of 28.5 (SD \pm 5.1). Cardiovascular disease, chronic lung disease and diabetes mellitus were the most noted comorbidities in the medical history. More than two-third of the study population needed oxygen suppletion at ED presentation and 435 patients (61.5\%) received (hydroxy)chloroquine as COVID-19-specific treatment. The median MEWS at the ED was 2.0 (IQR 2.0-4.0).

Table 1. Baseline characteristics ${ }^{\text {a }}$.

\begin{tabular}{|c|c|c|c|c|}
\hline \multirow[t]{2}{*}{ Characteristic } & \multirow[b]{2}{*}{$\begin{array}{c}\text { Total } \\
n=629\end{array}$} & \multicolumn{2}{|c|}{ Previous Coxiella burnetii Infection } & \multirow[t]{2}{*}{$p$-Value ${ }^{b}$} \\
\hline & & $\begin{array}{c}\text { With } \\
n=117(\mathbf{1 8 . 6 \% )})\end{array}$ & $\begin{array}{c}\text { Without } \\
n=512(81.4 \%)\end{array}$ & \\
\hline Age in years (mean, SD) & $68.0 \pm 12.5$ & $67.46 \pm 11.2$ & $68.07 \pm 12.7$ & 0.635 \\
\hline Male sex (n, \%) & $412(65.5 \%)$ & $83(70.9 \%)$ & $329(64.3 \%)$ & 0.170 \\
\hline uCCI (median, IQR) & $0.0(0.0-1.0)$ & $0.0(0.0-1.0)$ & $0.0(0.0-1.0)$ & 0.579 \\
\hline BMI (mean, SD) & $28.5 \pm 5.1$ & $29.27 \pm 5.0$ & $28.35 \pm 5.1$ & 0.090 \\
\hline
\end{tabular}


Table 1. Cont.

\begin{tabular}{|c|c|c|c|c|}
\hline \multirow[t]{2}{*}{ Characteristic } & \multirow[b]{2}{*}{$\begin{array}{c}\text { Total } \\
n=629\end{array}$} & \multicolumn{2}{|c|}{ Previous Coxiella burnetii Infection } & \multirow[t]{2}{*}{$p$-Value } \\
\hline & & $\begin{array}{c}\text { With } \\
n=117(18.6 \%)\end{array}$ & $\begin{array}{c}\text { Without } \\
n=512(81.4 \%)\end{array}$ & \\
\hline \multicolumn{5}{|l|}{ Medical history: } \\
\hline Cardiovascular disease $(\mathrm{n}, \%)$ & $250(39.7 \%)$ & $52(44.4 \%)$ & $198(38.7 \%)$ & 0.250 \\
\hline Chronic lung disease (n, \%) & $111(17.6 \%)$ & $23(19.7 \%)$ & $88(17.2 \%)$ & 0.527 \\
\hline Diabetes mellitus (n, \%) & $141(22.4 \%)$ & $30(25.6 \%)$ & $111(21.7 \%)$ & 0.354 \\
\hline Immunocompromised state $(n, \%)$ & $72(11.4 \%)$ & $9(7.7 \%)$ & $63(12.3 \%)$ & 0.123 \\
\hline Lymphoma (n, \%) & $9(1.4 \%)$ & $0(-)$ & $9(1.8 \%)$ & 0.222 \\
\hline Leukemia (n, \%) & $10(1.6 \%)$ & $4(3.4 \%)$ & $6(1.2 \%)$ & 0.157 \\
\hline Malignancy (n, \%) & $85(13.5 \%)$ & $18(15.4 \%)$ & $67(13.1 \%)$ & 0.512 \\
\hline With metastasis (n, \%) & $8(2.5 \%)$ & $2(1.7 \%)$ & $6(1.2 \%)$ & 0.646 \\
\hline Nursing home (n, \%) & $16(2.5 \%)$ & $3(2.6 \%)$ & $13(2.5 \%)$ & 1.000 \\
\hline \multicolumn{5}{|l|}{ ED presentation } \\
\hline Oxygen suppletion (n, \%) & $435(69.2 \%)$ & $79(67.5 \%)$ & $356(69.5 \%)$ & 0.671 \\
\hline MEWS (median, IQR) & $2.0(2.0-4.0)$ & $2.0(2.0-4.0)$ & $2.0(1.0-4.0)$ & 0.796 \\
\hline (Hydroxy)chloroquine treatment $(n, \%)$ & $387(61.5 \%)$ & $75(64.1 \%)$ & $312(60.9 \%)$ & 0.526 \\
\hline \multicolumn{5}{|l|}{ Complaints at presentation } \\
\hline Dyspnea (n, \%) & $441(70.1 \%)$ & $93(79.5 \%)$ & $348(68.0 \%)$ & 0.014 \\
\hline Cough (n, \%) & $411(65.3 \%)$ & $76(65.0 \%)$ & $335(65.4 \%)$ & 0.932 \\
\hline Thoracic pain $(\mathrm{n}, \%)$ & $59(9.4 \%)$ & $10(8.5 \%)$ & $49(9.6 \%)$ & 0.732 \\
\hline
\end{tabular}

Abbreviations: n: number, SD: standard deviation, uCCI: updated Charlson comorbidity index, IQR: inter quartile range, BMI: body mass index, ED: emergency department, MEWS: modified early warning score. ${ }^{a}$ Binary variables are presented as absolute numbers and percentages, continuous variables are presented as mean with standard deviation for normally distributed variables and median with interquartile range (IQR) for the nonnormally distributed. ${ }^{b} p$-values were calculated using Pearson's chi square test or Fisher's Exact test to compare proportions between groups. An independent sample $t$-test was used to compare means for normally distributed continuous variables and a Mann-Whitney U test for non-normally distributed continuous variables.

\subsection{Outcome}

In total, 126 patients $(20.0 \%)$ were admitted to the ICU and 156 COVID-19 patients (24.8\%) died in the hospital. Overall, the combined primary outcome, admission to the ICU and/or in-hospital mortality, occurred in 254 patients (40.4\%). Among patients with and without previous C. burnetii infection, the primary outcome occurred in $40.2 \%$ and $40.4 \%$ of cases, respectively (adjusted OR of 0.926 (95\% CI 0.605-1.416)). A total of 578 patients (91.9\%) were admitted to a regular ward (regardless of ICU admission at any point). Among patients with and without previous $C$. burnetii infection, the adjusted OR for the secondary outcomes in-hospital mortality $(22.2 \%$ vs. $25.4 \%)$, ICU admission ( $24.8 \%$ vs. $19.7 \%)$ and regular ward admission $(89.7 \%$ vs. $92.4 \%)$ were 0.825 (95\% CI $0.488-1.393)$, 1.299 (95\% CI 0.799-2.112) and 0.612 (95\% CI 0.303-1.237), respectively (Table 2). 
Table 2. Risk of in-hospital mortality and ICU admission ${ }^{\text {a }}$.

\begin{tabular}{|c|c|c|c|c|}
\hline & \multicolumn{4}{|c|}{ Previous Coxiella burnetii Infection } \\
\hline & With $(n=117)$ & Without $(n=512)$ & OR $(95 \% \mathrm{CI})$ & 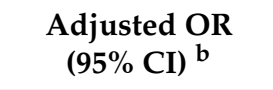 \\
\hline Combined primary outcome & $47(40.2)$ & $207(40.4)$ & $0.989(0.657-1.490)$ & $0.926(0.605-1.416)$ \\
\hline In-hospital mortality & $26(22.2)$ & $130(25.4)$ & $0.840(0.520-1.356)$ & $0.825(0.488-1.393)$ \\
\hline ICU admission & $29(24.8)$ & $101(19.7)$ & $1.341(0.836-2.152)$ & $1.299(0.799-2.112)$ \\
\hline Ward admission & $105(89.7)$ & $473(92.4)$ & $0.721(0.365-1.425)$ & $0.612(0.303-1.237)$ \\
\hline
\end{tabular}

\section{Discussion}

We found no decreased or increased risk of mortality, ICU admission, and/or admission to a regular ward after previous C. burnetii infection in patients admitted to the ED with COVID-19. Only one other study has been published assessing a possible association between previous $C$. burnetii infection and COVID-19 in an area with high $Q$ fever incidence. C. burnetii seroprevalence was assessed in 50 Dutch patients admitted to the hospital with COVID-19. A slightly increased seroprevalence of C. burnetii IgG of $16.0 \%$ was found in these patients compared to seroprevalence in blood donors (12.2\%) [26], and patients with a vascular of cardiac heart valve surgery in that area (14.9\%). C. burnetii IgG seroprevalence among COVID-19 patients that were admitted to the ICU or died, was $15.7 \%$ and $9.0 \%$, respectively. No clear association was found between previous $C$. burnetii infection and an adverse outcome of COVID-19 infection [27].

In our study, $18.6 \%$ of the COVID-19 patients had evidence of previous C. burnetii infection. However, this percentage cannot be compared directly to seroprevalence studies $[2,26]$. The percentages in seroprevalence studies are based solely on C. burnetii serology results at a certain time point. In our study, the percentage of patients with evidence of previous $C$. burnetii infection is based on both seroprevalence, and on earlier performed C. burnetii serology and PCR results on diagnostic samples extracted from the EHR. However, long term follow-up of acute $Q$ fever patients showed loss of seropositivity (1.1\% to $23.4 \%)$ some years after $\mathrm{Q}$ fever infection [28-31]. Therefore, the percentage of patients with evidence of previous C. burnetii infection in our study can be a slight overestimation because of the patients included based upon extra information from the EHR, or it can be underestimation because of possible loss of seropositivity in the long time period between the end of the Dutch $Q$ fever and the start of the COVID-19 outbreak, compared to the seroprevalence studies.

The association between adverse outcomes after COVID-19 and several other infectious diseases is studied. Although immune dysregulation is described in patients infected with both SARS-CoV-2 and HBV, a co-infection did not significantly affect the outcome of COVID-19 [12,32]. An association between past CMV infection and adverse outcomes after infection with SARS-CoV-2 is hypothesized through increase of inflammatory mediated cytokines in these patients [13,33], however, a direct association study has not been performed to this date. A systematic review in people living with HIV described increased susceptibility to SARS-CoV-2 infection and a higher mortality [34].

Our population consisted only of patients who had visited the ED, a population that does not represent the whole population of COVID-19 patients. Consequently, we could not identify a possible protective or adverse effect of previous C. burnetii on the course of SARS-CoV-2 infection prior to presentation at the ED. All conclusions only apply to COVID-19 patients, given they were referred to the ED. However, in view of the relative high percentage of patients with evidence of previous $C$. burnetii infection presented at the 
ED, it could suggest that previous $C$. burnetii infection causes an increased susceptibility to SARS-CoV-2 and/or increases the chance of referral to the ED with COVID-19.

In the case that previous C. burnetii infection plays no role in the course of COVID-19 after ED presentation, the question arises which factors might be responsible for the large outbreak in this region at the start of the Dutch epidemic. The southern region of the Netherlands was the part of the country that had the earliest spring break period in 2020 and many inhabitants went skiing in Italy where SARS-CoV-2 was already spreading. Hence, travel to this region was advised against short after. This holiday-period coincided with the Carnival festival, which is a popular regional yearly four-day festival in the southern region during which many inhabitants come together in city centers and bars. Possibly, introduction from Italy and spread through Carnival could be an explanation for the initial emergence of SARS-CoV-2 in this region. Another possible explanation for the high incidence of COVID-19 is the high number of livestock farms in this region. The air quality around livestock farms is associated with higher risk of respiratory tract infection [35-37].

Furthermore, in literature, diet and nutrition status is proposed as having a potential impact on COVID-19 outcomes [38]. However, this will probably play no small role in our study because all patients are from the same region and there is no reason to assume the diet is different between the group of patients with and without previous C. burnetii infection.

Strengths of our research are the high number of patients with evidence of previous C. burnetii infection, which makes this population ideal to study the association between $Q$ fever and COVID-19. The analysis was corrected for potential confounders, taking in mind that comorbidities diagnosed after $C$. burnetii infection cannot be a confounder and should not be corrected for. The data collected from the EHR was complete apart from information on patient height or weight, which were handled by multiple imputations.

Our research harbors several limitations. Firstly, the design of our study was of a retrospective nature; this can potentially introduce unmeasured confounders. As all data had to be extracted from the EHR, the confounders were limited to the date available within in the EHR. Secondly, a loss of seropositivity after acute $Q$ fever is mentioned earlier. This can lead to misclassification of the determinant because patients with a previous C. burnetii infection could be classified as without infection. Misclassification of the determinant possibly leads to underestimation of the possible effect of the association. However, identifying previous C. burnetii infection through the EHR lowers the risk of misclassification in this study. In addition, patients were excluded from the analysis when no information on evidence of a previous $C$ burnetii infection could be determined from patient history and no blood sample was stored. It is possible that more blood samples were stored from patients who had adverse outcomes. Therefore, these patients could be 'missing not at random' and potentially induce selection bias. We performed two sensitivity analyses testing the robustness of the estimates found in our initial analysis. First, an analysis excluding patients with evidence of previous C. burnetii infection determined through analysis of stored blood samples. Second, an analysis excluding patients with evidence of previous C. burnetii infection determined from the EHR. This way the analysis is separately performed for patients with a 'symptomatic' C. burnetii infection and those with an 'asymptomatic' C. burnetii infection. These sensitivity analyses did not change our initial findings. Lastly, the confidence interval around the estimate of the primary outcome is fairly broad. With the current sample size and ratio between the two groups a significant difference in risk of the combined primary outcome of $\pm 15 \%$ could be detected. A total sample size of 1280 and 5000 COVID-19 patients is needed to detect a difference in risk of the combined primary outcome of $10 \%$ and $5 \%$, respectively. However, the differences in adverse outcomes after SARS-CoV-2 infection in patients with and without previous C. burnetii infection are so small that the detection of a clinically relevant difference with a larger sample size is not expected. In conclusion, we report no association between 
previous C. burnetii infection and risk of ICU admission and/or mortality for COVID-19 patients visiting the ED.

Author Contributions: J.M.W. contributed to conceptualization, funding acquisition, data curation, formal analysis, methodology, project administration, visualization, writing—original draft, and review and editing. R.v.S. contributed to data curation, formal analysis, methodology, visualization, writing-original draft, and review and editing. C.P.B.-R. and A.I.M.H., contributed to conceptualization, funding acquisition, formal analysis, methodology, writing - review and editing, and supervision. P.C.W. and J.J.O. contributed to conceptualization, funding acquisition, formal analysis, methodology, project administration, writing - review and editing, and supervision. All authors have read and agreed to the published version of the manuscript.

Funding: This work was supported financially by the National Institute for Public Health and Environment (RIVM) and Jeroen Bosch Hospital in 's-Hertogenbosch. The funder had no role in study design, data collection, data analysis, data interpretation, or writing of the report.

Institutional Review Board Statement: The study was conducted in accordance with the Declaration of Helsinki. The local medical ethics committee stated that this study needed no specific review.

Informed Consent Statement: The Internal Review Board of the Jeroen Bosch Hospital and Bernhoven approved the use of remnant clinical samples and waived the need for informed consent from patients.

Data Availability Statement: The data presented in this study are available on request from the corresponding author. The data are not publicly available due to privacy reasons.

Conflicts of Interest: The authors declare no conflict of interest.

\section{References}

1. Maurin, M.; Raoult, D. Q fever. Clin. Microbiol. Rev. 1999, 12, 518-553. [CrossRef] [PubMed]

2. Kampschreur, L.M.; Hagenaars, J.C.J.P.; Wielders, C.C.H.; Elsman, P.; Lestrade, P.J.; Koning, O.H.J.; Oosterheert, J.J.; Renders, N.H.M.; Wever, P.C. Screening for Coxiella burnetii seroprevalence in chronic $Q$ fever high-risk groups reveals the magnitude of the Dutch Q fever outbreak. Epidemiol. Infect. 2013, 141, 847-851. [CrossRef] [PubMed]

3. Dijkstra, F.; van der Hoek, W.; Wijers, N.; Schimmer, B.; Rietveld, A.; Wijkmans, C.J.; Vellema, P.; Schneeberger, P.M. The 2007-2010 $Q$ fever epidemic in the Netherlands: Characteristics of notified acute $Q$ fever patients and the association with dairy goat farming. FEMS Immunol. Med. Microbiol. 2012, 64, 3-12. [CrossRef] [PubMed]

4. Morroy, G.; Keijmel, S.P.; Delsing, C.E.; Bleijenberg, G.; Langendam, M.; Timen, A.; Bleeker-Rovers, C.P. Fatigue following acute Q-fever: A systematic literature review. PLoS ONE 2016, 11, e0155884. [CrossRef]

5. Van Roeden, S.E.; Wever, P.C.; Kampschreur, L.M.; Gruteke, P.; van der Hoek, W.; Hoepelman, A.I.M.; Bleeker-Rovers, C.P.; Oosterheert, J.J. Chronic Q fever-related complications and mortality: Data from a nationwide cohort. Clin. Microbiol. Infect. 2019, 25, 1390-1398. [CrossRef]

6. Alderweireld, C.E.A.; Buiting, A.G.M.; Murk, J.L.A.N.; Verweij, J.J.; Berrevoets, M.A.H.; van Kasteren, M.E.E. COVID-19: Patient zero in the Netherlands. Ned. Tijdschr. Geneeskd. 2020, 164, D4962.

7. Reusken, C.B.; Buiting, A.; Bleeker-Rovers, C.; Diederen, B.; Hooiveld, M.; Friesema, I.; Koopmans, M.; Kortbeek, T.; Lutgens, S.P.M.; Meijer, A.; et al. Rapid assessment of regional SARS-CoV-2 community transmission through a convenience sample of healthcare workers, the Netherlands, March 2020. Eurosurveillance 2020, 25, 6-9. [CrossRef]

8. Rao, K.S.; Suryaprakash, V.; Senthilkumar, R.; Preethy, S.; Katoh, S.; Ikewaki, N.; Abraham, S.J.K. Role of Immune Dysregulation in Increased Mortality Among a Specific Subset of COVID-19 Patients and Immune-Enhancement Strategies for Combatting Through Nutritional Supplements. Front. Immunol. 2020, 11, 1-9. [CrossRef]

9. Roberts, J.; Pritchard, A.L.; Treweeke, A.T.; Rossi, A.G.; Brace, N.; Cahill, P.; MacRury, S.M.; Wei, J.; Megson, I.L. Why Is COVID-19 More Severe in Patients With Diabetes? The Role of Angiotensin-Converting Enzyme 2, Endothelial Dysfunction and the Immunoinflammatory System. Front. Cardiovasc. Med. 2021, 7, 1-23. [CrossRef]

10. Costagliola, G.; Spada, E.; Consolini, R. Age-related differences in the immune response could contribute to determine the spectrum of severity of COVID-19. Immunity, Inflamm. Dis. 2021, 9, 331-339. [CrossRef]

11. Korakas, E.; Ikonomidis, I.; Kousathana, F.; Balampanis, K.; Kountouri, A.; Raptis, A.; Palaiodimou, L.; Kokkinos, A.; Lambadiari, V. Obesity and COVID-19: Immune and metabolic derangement as a possible link to adverse clinical outcomes. Am. J. Physiol.-Endocrinol. Metab. 2020, 319, E105-E109. [CrossRef]

12. Liu, R.; Zhao, L.; Cheng, X.; Han, H.; Li, C.; Li, D.; Liu, A.; Gao, G.; Zhou, F.; Liu, F.; et al. Clinical characteristics of COVID-19 patients with hepatitis B virus infection-A retrospective study. Liver Int. 2021, 41, 720-730. [CrossRef]

13. Poloni, C.; Szyf, M.; Cheishvili, D.; Tsoukas, C.M. Are the healthy vulnerable? Cytomegalovirus seropositivity in healthy adults is associated with accelerated epigenetic age and immune-dysregulation. J Infect Dis 2021, preprint. [CrossRef] 
14. Raijmakers, R.P.H.; Jansen, A.F.M.; Keijmel, S.P.; van der Meer, J.W.M.; Joosten, L.A.B.; Netea, M.G.; Bleeker-Rovers, C.P. A possible link between recurrent upper respiratory tract infections and lower cytokine production in patients with $Q$ fever fatigue syndrome. Eur. J. Immunol. 2019, 49, 1015-1022. [CrossRef]

15. Raijmakers, R.P.; Stenos, J.; Keijmel, S.P.; Ter Horst, R.; Novakovic, B.; Nguyen, C.; Van Der Meer, J.W.; Netea, M.G.; BleekerRovers, C.P.; Joosten, L.A.; et al. Long-Lasting Transcriptional Changes in Circulating Monocytes of Acute Q Fever Patients. Open Forum Infect. Dis. 2019, 6, 1-9. [CrossRef]

16. Netea, M.G.; Giamarellos-Bourboulisi, E.J.; Domínguez-Andrés, J.; Curtis, N.; van Crevel, R.; van de Veerdonk, F.L.; Bonten, M.J.M. Trained Immunity: A Tool for Reducing Susceptibility to and the Severity of SARS-CoV-2 Infection. Cell 2020, 181, 969-977. [CrossRef]

17. Netea, M.G.; Joosten, L.A.B.; Latz, E.; Mills, K.H.G.; Natoli, G.; Stunnenberg, H.G.; O’Neill, L.A.J.; Xavier, R.J. Trained immunity: A program of innate immune memory in health and disease. Science 2016, 352, 427. [CrossRef]

18. Ohrui, T.; Nakayama, K.T.F.; Chiba, H.; Sasaki, H. Prevention of elderly pneumonia by pneumococcal, influenza and BCG vaccinations. Nihon Ronen Igakkai Zasshi 2005, 42, 34-36. [CrossRef]

19. Wardhana; Datau, E.A.; Sultana, A.; Mandang, V.V.; Jim, E. The efficacy of Bacillus Calmette-Guerin vaccinations for the prevention of acute upper respiratory tract infection in the elderly. Acta Med. Indones. 2011, 43, 185-190.

20. Giamarellos-Bourboulis, E.J.; Tsilika, M.; Moorlag, S.; Antonakos, N.; Kotsaki, A.; Domínguez-Andrés, J.; Kyriazopoulou, E.; Gkavogianni, T.; Adami, M.E.; Damoraki, G.; et al. Activate: Randomized Clinical Trial of BCG Vaccination against Infection in the Elderly. Cell 2020, 183, 315-323.e9. [CrossRef]

21. Ten Doesschate, T.; Moorlag, S.J.C.F.M.; Van Der Vaart, T.W.; Taks, E.; Debisarun, P.; Ten Oever, J.; Bleeker-Rovers, C.P.; Verhagen, P.B.; Lalmohamed, A.; Ter Heine, R.; et al. Two Randomized Controlled Trials of Bacillus Calmette-Guérin Vaccination to reduce absenteeism among health care workers and hospital admission by elderly persons during the COVID-19 pandemic: A structured summary of the study protocols for two randomised. Trials 2020, 21, 20-21. [CrossRef]

22. Madsen, A.M.R.; Schaltz-Buchholzer, F.; Benfield, T.; Bjerregaard-Andersen, M.; Dalgaard, L.S.; Dam, C.; Ditlev, S.B.; Faizi, G.; Johansen, I.S.; Kofoed, P.E.; et al. Using BCG vaccine to enhance non-specific protection of health care workers during the COVID-19 pandemic: A structured summary of a study protocol for a randomised controlled trial in Denmark. Trials 2020, 21, 20-22. [CrossRef]

23. Junqueira-Kipnis, A.P.; dos Anjos, L.R.B.; de Souza Barbosa, L.C.; da Costa, A.C.; Borges, K.C.M.; da Rocha Oliveira Cardoso, A.; Ribeiro, K.M.; Rosa, S.B.A.; de Castro Souza, C.; das Neves, R.C.; et al. BCG revaccination of health workers in Brazil to improve innate immune responses against COVID-19: A structured summary of a study protocol for a randomised controlled trial. Trials 2020, 21, 20-22. [CrossRef]

24. Quan, H.; Li, B.; Couris, C.M.; Fushimi, K.; Graham, P.; Hider, P.; Januel, J.M.; Sundararajan, V. Updating and validating the charlson comorbidity index and score for risk adjustment in hospital discharge abstracts using data from 6 countries. Am. J. Epidemiol. 2011, 173, 676-682. [CrossRef] [PubMed]

25. Subbe, C.P.; Kruger, M.; Rutherford, P.; Gemmel, L. Validation of a modified Early Warning Score in medical admissions. QJM 2001, 94, 512-526. [CrossRef] [PubMed]

26. Hogema, B.M.; Slot, E.; Molier, M.; Schneeberger, P.M.; Hermans, M.H.; Van Hannen, E.J.; Van Der Hoek, W.; Cuijpers, H.T.; Zaaijer, H.L. Coxiella burnetii infection among blood donors during the 2009 Q-fever outbreak in The Netherlands. Transfusion 2012, 52, 144-150. [CrossRef] [PubMed]

27. Mulder, B.; Walk, J.; Dautzenberg, M.; Lohuis, A.O.; Nabuurs, M.; Dofferhoff, T. Doorgemaakte Q-koorts geassocieerd met ernstige ziekte of sterfte van COVID-19-patiënten? Ned. Tijdschr. Med. Microbiol. 2020, 28, 157-160.

28. Hussain-Yusuf, H.; Islam, A.; Healy, B.; Lockhart, M.; Nguyen, C.; Sukocheva, O.; Stenos, J.; Graves, S. An analysis of Q fever patients 6 years after an outbreak in Newport, Wales, UK. QJM 2012, 105, 1067-1073. [CrossRef]

29. Wielders, C.C.H.; Van Loenhout, J.A.F.; Morroy, G.; Rietveld, A.; Notermans, D.W.; Wever, P.C.; Renders, N.H.M.; Leenders, A.C.A.P.; Van Der Hoek, W.; Schneeberger, P.M. Long-term Serological follow-up of acute Q-fever patients after a large epidemic. PLoS ONE 2015, 10, e0131848. [CrossRef]

30. Morroy, G.; Van Der Hoek, W.; Albers, J.; Coutinho, R.A.; Bleeker-Rovers, C.P.; Schneeberger, P.M. Population screening for chronic Q-fever seven years after a major outbreak. PLoS ONE 2015, 10, e0131777. [CrossRef]

31. Buijs, S.B.; Stuart, S.K.; Oosterheert, J.J.; Karhof, S.; Hoepelman, A.I.M.; Renders, N.H.M.; van Petersen, A.S.; Bleeker-Rovers, C.P.; Wever, P.C.; Koning, O.H.J. Long-term serological follow-up after primary Coxiella burnetii infection in patients with vascular risk factors for chronic Q fever. Eur. J. Clin. Microbiol. Infect. Dis. 2021, 40, 1569-1572. [CrossRef]

32. Bekçibaşı, M.; Arslan, E. Severe acute respiratory syndrome coronavirus 2 (SARS-CoV-2)/Hepatitis B virus (HBV) Co-infected Patients: A case series and review of the literature. Int. J. Clin. Pract. 2021, 75, e14412. [CrossRef]

33. Kadambari, S.; Klenerman, P.; Pollard, A.J. Why the elderly appear to be more severely affected by COVID-19: The potential role of immunosenescence and CMV. Rev. Med. Virol. 2020, 30, 1-5. [CrossRef]

34. Ssentongo, P.; Heilbrunn, E.S.; Ssentongo, A.E.; Advani, S.; Chinchilli, V.M.; Nunez, J.J.; Du, P. Epidemiology and outcomes of COVID-19 in HIV-infected individuals: A systematic review and meta-analysis. Sci. Rep. 2021, 11, 1-12. [CrossRef]

35. Post, P.M.; Hogerwerf, L.; Huss, A.; Petie, R.; Boender, G.J.; Baliatsas, C.; Lebret, E.; Heederik, D.; Hagenaars, T.J.; IJzermans, C.J.; et al. Risk of pneumonia among residents living near goat and poultry farms during 2014-2016. PLoS ONE 2019, 14, e0223601. [CrossRef] 
36. Kalkowska, D.A.; Boender, G.J.; Smit, L.A.M.; Baliatsas, C.; Yzermans, J.; Heederik, D.J.J.; Hagenaars, T.J. Associations between pneumonia and residential distance to livestock farms over a five-year period in a large population-based study. PLoS ONE 2018, 13, e0200813. [CrossRef]

37. O'Connor, A.M.; Auvermann, B.W.; Dzikamunhenga, R.S.; Glanville, J.M.; Higgins, J.P.T.; Kirychuk, S.P.; Sargeant, J.M.; Totton, S.C.; Wood, H.; Von Essen, S.G. Updated systematic review: Associations between proximity to animal feeding operations and health of individuals in nearby communities. Syst. Rev. 2017, 6, 1-20. [CrossRef]

38. Skrajnowska, D.; Brumer, M.; Kankowska, S.; Matysek, M.; Miazio, N.; Bobrowska-Korczak, B. COVID 19: Diet composition and health. Nutrients 2021, 13, 2980. [CrossRef] 